\title{
Electrochemical Capacitance of Activated Carbons Regenerated using Thermal and Chemical Activation
}

\author{
Jung Eun Park ${ }^{1}$, Gi Bbum Lee ${ }^{1}$, and Sang Youp Hwang ${ }^{2 *}$ \\ ${ }^{1}$ Bio Resource Center, Institute for Advanced Engineering, Yongin 17180, Korea \\ ${ }^{2}$ Department of Energy Engineering, Konkuk University, 120 Neungdong-ro, Gwangjin-gu, Seoul 05029, Korea
}

\begin{abstract}
Spent activated carbons (SACs) collected from a water treatment plant were regenerated and then adopted as electrochemical material in capacitors. The SACs used in this study were regenerated via two steps, namely thermal and chemical activation. However, during the activation process, the adsorbates were converted into ashes, which caused pore blockage and decreased specific surface area. The regenerated SACs were washed with acid solutions with different levels of acidity (strong: $\mathrm{HCl}$, mild: $\mathrm{H}_{3} \mathrm{PO}_{4}$, and weak: $\mathrm{H}_{2} \mathrm{O}_{2}$ ) to remove the ashes. The regenerated SACs washed with $\mathrm{HCl}$ exhibited the highest specific surface area, although their capacitance was not the highest. Conversely, the specific surface area of regenerated SACs washed using $\mathrm{H}_{3} \mathrm{PO}_{4}$ was slightly lower than that of $\mathrm{HCl}$, but exhibited higher capacitance and electrochemical stability. Although the strong acid removed the generated ashes in the pores efficiently, it could adversely affect their structural stability, which would lead to lower capacitance.
\end{abstract}

Keywords : Activated Carbon, Spent Activated Carbon, Regeneration, Electrochemical Material, Capacitance

Received : 30 November 2020, Accepted : 23 February 2021

\section{Introduction}

Activated carbons (ACs) have been used in pollution remediation in both liquid and gas phase effluent from industries [1]. The pollutants are usually adsorbed onto AC surfaces before being released into the atmosphere or water, which prevents environmental pollution [1-3]. ACs are usually disposed in landfills or thermally regenerated when they lose their adsorption capacity [4,5]. For example, according to previous reports, over $70 \%$ of spent ACs (SACs) generated by the industrial complex (Yeo-su) in South Korea are disposed in landfill sites [6]. While fresh ACs are used in pollutant remediation, SACs could lead to secondary environmental pollution, such as soil poisoning $[3,6]$.

The potential reuse of SACs following regeneration has attracted the attention of environmental researchers $[6,7]$. To date, $\mathrm{SAC}$ regeneration has been

*E-mail address: syhwang80@gmail.com

DOI: https://doi.org/10.33961/jecst.2020.01683

This is an open-access article distributed under the terms of the Creative Commons Attribution Non-Commercial License (http://creativecommons.org/licenses/by-nc/4.0) Attribution Non-Commercial License (http://creativecommons.org/licenses/by-nc/4.0)
which permits unrestricted non-commercial use, distribution, and reproduction in any which permits unrestricted non-commercial use, distri
medium, provided the original work is properly cited. studied extensively. SACs could be regenerated using various approaches, such as thermal and chemical treatments, and chemical activation [8]. Among the methods, thermal regeneration has been applied extensively due to its relative simplicity and low associated costs $[9,10]$.

SACs can be classified into two categories based on whether they remove pollutants in the 1) gas or 2) liquid phase. ACs have been used widely to remove gas pollutants such as volatile organic compounds (VOCs). The VOCs adsorbed on the ACs are removed or decomposed efficiently by thermal treatment, and then ACs regenerated [6]. However, ACs used in removal of pollutants from water require relatively high energy levels due to the associated high latent heat of vaporization, in addition to the relatively complex regeneration process required [11]. Specifically, ACs adsorb various metallic ions in water, and then the metal ions are converted into ash during thermal regeneration (as illustrated in Fig. 1) [12]. The thermal regeneration process evaporates the moisture; however, the adsorbed metal ions cannot be eliminated and they remain in the pores $[4,13,14]$, which decreases their surface area. Conse- 


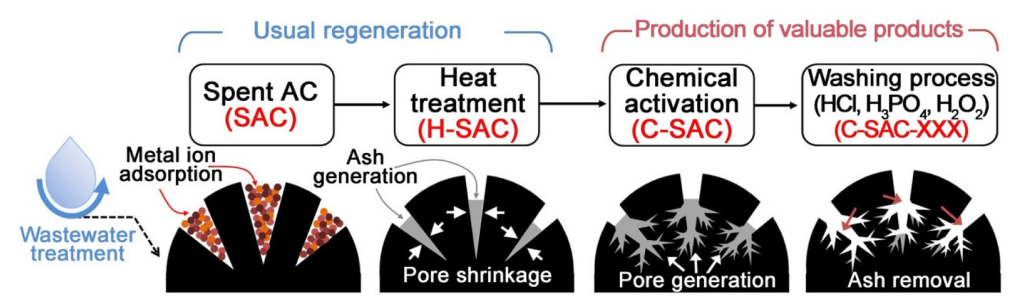

Fig. 1. SAC regeneration using thermal and chemical activation.

quently, an additional process is required to remove the ash prior to the use of regenerated ACs.

A previous report has already highlighted the limitations of thermal regeneration with regard to SACs. Han et al. regenerated ACs using the three methods, including heat treatment, ultrasonication, and solvent extraction [13]. Both ultrasonication and solvent extraction methods exhibited regeneration rates superior to that of thermal regeneration because coke deposits on micropores under thermal regeneration were eliminated. In addition, Sabio et al. recommended thermal regeneration using gasifying agents (carbon dioxide and air) to facilitate complete regeneration [4]. The SACs thermally regenerated using the gasifying agents had increased micropores, performing the initial capacity. Bagreev et al. also regenerated SACs by heating in the atmosphere [14]. The thermal treatment using the air removed residues in the pores, and the results exhibited $100 \%$ pore regeneration. As aforementioned, the previous reports also extensively explored pore blockage caused by ashes and coke through thermal treatment, because the removal of ash from pores is a key aspect of SAC regeneration.

In the present study, the SACs were thermally regenerated, and then chemically activated, according to the schematic presented in Fig. 1. Chemical activation often enhances AC surface area, after which they could be used in various applications. The regenerated ACs using the only thermal treatment was limited to the application because of the generated ashes and decreased surface areas. Therefore, in previous studies, regenerated ACs have been mostly reused for pollutant removal. However, the ACs regenerated in the present study using both thermal and chemical activation methods had relatively high surface areas, so that they could be applied in electrochemical materials such as capacitors. In addition, the ashes generated on the regenerated ACs follow- ing both treatments were removed efficiently using various acid treatments. The relationship between ash removal and surface area was investigated, in addition to the pores created by ash removal. Furthermore, the electrochemical properties of the ACs were also investigated. The electrochemical properties of the ACs produced using both the thermal and chemical activations were enhanced, and the properties were also superior to those of the ACs regenerated using the previous method, such as thermal or chemical treatment. The regeneration method that is introduced in the present study could be a new guideline for $\mathrm{SAC}$ regeneration and reuse.

\section{Materials and Methods}

\subsection{Regeneration of spent ACs (SACs)}

SACs were collected from a water purification plant in Gyeonggi-do Province, South Korea. The collected SACs were dried in an oven at $100^{\circ} \mathrm{C}$ overnight prior to use. The dried SACs were thermally regenerated at $500^{\circ} \mathrm{C}$ for $10 \mathrm{~h}$. The thermally regenerated SACs are hereafter referred to as H-SACs. In addition, the H-SACs were chemically regenerated using the $95 \%$ potassium hydroxide $(\mathrm{KOH})$ (Samchun Chem. Co., Korea). First, the H-SACs were mixed with $\mathrm{KOH}$ at a weight ratio of $1: 2$ (H-ACs: $\mathrm{KOH}=1: 2$ ), and then the mixture was placed in a vertical furnace. The sample mixtures in the furnaces were heated gradually until $750^{\circ} \mathrm{C}$, with heat ramped up at a rate of $5^{\circ} \mathrm{C} / \mathrm{min}$. The temperature was maintained at $750^{\circ} \mathrm{C}$ for $1 \mathrm{~h}$. After the $\mathrm{KOH}$ treatment process, the temperature was increased again up to $850^{\circ} \mathrm{C}$, where it was maintained for $3 \mathrm{~h}$ [15].

The chemically activated H-SACs are hereafter referred to as C-SACs. The C-SACs were treated with three acid solutions to remove the ashes produced. Hydrogen chloride (35\%) ( $\mathrm{HCl}$, Samchun Chem. Co.), $85.0 \%$ phosphoric acid $\left(\mathrm{H}_{3} \mathrm{PO}_{4}\right.$, Sigma 
Aldrich), and $34.5 \%$ hydrogen peroxide $\left(\mathrm{H}_{2} \mathrm{O}_{2}\right.$, Samchun Chem. Co.) were used for the acid treatments. After chemical activation, the samples were washed using tap water and then treated with $0.5 \mathrm{M}$ of each acid solution. The samples treated with the acids are hereafter referred to as C-SAC-treated acids (for example, C$\mathrm{SAC}-\mathrm{HCl}$ was treated with $\mathrm{HCl}$ solution).

\subsection{Characterization of prepared ACs}

The surface areas and pore distributions of prepared samples (SAC, H-SAC, C-SAC-HCl, C-SAC$\mathrm{H}_{3} \mathrm{PO}_{4}$, and C-SAC- $\mathrm{H}_{2} \mathrm{O}_{2}$ ) were analyzed using the Brunauer-Emmett-Teller and Barret-Joyner-Halenda $(\mathrm{BJH})$ method based on the nitrogen adsorption obtained at $77 \mathrm{~K}$ using a TriStar II (Micromeritics, USA) static volumetric analyser. Elemental analysis (EA) was conducted using an elemental analyser (FlashEA 1112 and FlashEA 2000, Thermo Fisher Scientific, Waltham, MA, USA) to determine the elemental concentrations (carbon, hydrogen, oxygen, nitrogen, and sulphur). For the proximate analysis, the samples were placed into a DF-4S furnace (Daeheung Science, Incheon, Korea). The samples were heated at $950^{\circ} \mathrm{C}$ for $7 \mathrm{~min}$, and then the temperature was decreased to $750^{\circ} \mathrm{C}$ for $10 \mathrm{~h}$. The ash, volatile matter, and fixed-carbon contents within the ACs were presented as percentages by weight. The content of carbon functional groups in the samples was confirmed via X-ray photoelectron spectroscopy (XPS, PHI-5000 VersaProbe (Ulvac-PHI)).

\subsection{Electrochemical capacitance measurement}

The capacitances of the samples were measured using the cyclic voltammetry (CV) method using a three-electrode system consisting of counter (platinum wire), reference ( $\mathrm{Ag} / \mathrm{AgCl}$ electrode), and working electrodes, linked to an electrochemical workstation (AUT302M, FRA2, Metrohm Autolab, Utrecht, The Netherlands). First, $10 \mathrm{mg}$ of AC powder was mixed with $0.1 \mathrm{~mL}$ of $5 \mathrm{wt} \%$ Nafion solution and $0.3 \mathrm{~mL}$ of $99.5 \%$ tert-Butanol (C4H10O, Daejung Chem. Co.), with brief sonication. Subsequently, $6.0 \mu \mathrm{L}$ of the prepared slurry was dropped onto a glassy carbon electrode, which acted as a working electrode [16]. Testing was conducted at different scan rates $(10-100 \mathrm{mV} / \mathrm{s})$ using a $0.5 \mathrm{M} \mathrm{H}_{2} \mathrm{SO}_{4}$ solution as an electrolyte. In addition, electrochemical stability was measured over 4000 cycles at a scan rate of $100 \mathrm{mV} / \mathrm{s}$ in the same electrolyte.

\section{Results and Discussion}

The SACs were regenerated sequentially by thermal and chemical activation processes, and then washed using three acid solutions. The thermal treatment process converted the adsorbed metal ions into ashes in the pores, which, in turn, decreased the surface areas of the SACs. A washing process is required to remove ashes before the utilisation of regenerated SACs. The acid solutions adopted for the washing processes were selected based on their acidities. As illustrated in Fig. S1, the acidity of the solutions (strong, mild, and weak) was classified based on pKa values [17-19]. The acidity of the acids used increased in the following order: $\mathrm{HCl}>\mathrm{H}_{3} \mathrm{PO}_{4}>$ $\mathrm{H}_{2} \mathrm{O}_{2}$, so that the $\mathrm{HCl}, \mathrm{H}_{3} \mathrm{PO}_{4}$, and $\mathrm{H}_{2} \mathrm{O}_{2}$ were strong, mild, and weak acids, respectively, were based on the pKa values.

Fig. 2 illustrates the relationship between ash contents and specific surface area. The specific surface areas of H-SACs were slightly higher than those of the other SACs, but their surface areas were still too low for them to be reused. The surface areas of $\mathrm{H}$ SACs were not fully recovered following thermal treatment alone. However, C-SACs, which were chemically activated and subsequently washed with acid solutions, exhibited higher specific surface areas than H-SACs and SACs. The reduction in ash content following acid washing increases SAC specific surface area, implying that surface area increased following chemical activation, and then the ashes generated from both the thermal and chemical activation processes were removed efficiently by acid treatment [3]. SAC surface area was as follows: C-SAC-

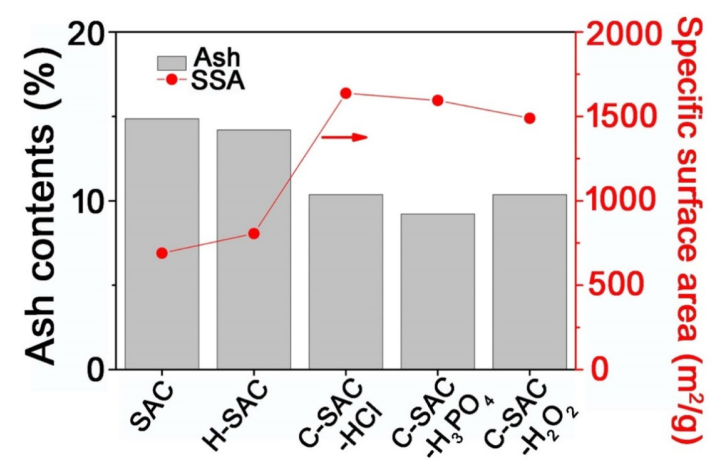

Fig. 2. Relationship between the residual ash contents and specific surface area 
$\mathrm{HCl}>\mathrm{C}-\mathrm{SAC}-\mathrm{H}_{3} \mathrm{PO}_{4}>\mathrm{C}-\mathrm{SAC}-\mathrm{H}_{2} \mathrm{O}_{2}$, indicating that the surface area increased with an increase in the acidity of the washing solution. The pore size distributions of samples were also shown in the Fig. S2. The pores of SAC and H-SAC were distributed between 3-4 nm. However, those pores were disappeared in the chemically regenerated SACs (C-SAC$\mathrm{HCl}, \mathrm{H}_{3} \mathrm{PO}_{4}$, and $\mathrm{H}_{2} \mathrm{O}_{2}$ ). This suggested that the pores shifted to lower smaller micropores $(<2 \mathrm{~nm})$, increasing the specific surface areas.

The electrochemical properties of the ACs prepared and commercial carbon (Vulcan XC-72) were investigated by $\mathrm{CV}$. The $\mathrm{CV}$ was conducted in $0.5 \mathrm{M}$ $\mathrm{H}_{2} \mathrm{SO}_{4}$ solution as an electrolyte at different scan rates (10-100 mV/s). Fig. 3 shows the $\mathrm{CV}$ curves and the calculated capacitance of SAC, H-SAC, C-SAC$\mathrm{HCl}, \mathrm{C}-\mathrm{SAC}-\mathrm{H}_{3} \mathrm{PO}_{4}, \mathrm{C}-\mathrm{SAC}-\mathrm{H}_{2} \mathrm{O}_{2}$, and Vulcan XC72. The specific capacitance of all the samples were calculated from $\mathrm{CV}$ curves using the following equation (1):

$$
\mathrm{C}_{\mathrm{s}}=\int \mathrm{IdV} /(\mathrm{m} \times \Delta \mathrm{V} \times \mathrm{S})
$$

where $\mathrm{C}_{\mathrm{s}}$ is the specific capacitance, $\int \mathrm{IdV}$ is the integrated area of the CV curve, $m$ is the mass of the ACs generated on the electrode, $\Delta \mathrm{V}$ is the potential window, and $\mathrm{S}$ is the potential scan rate [20,21].

As illustrated in Fig. 3a-c, the CV curves of samples exhibit a typical rectangular shape at various scan rates, and the current density increased with an increase in scan rate. In addition, the ACs that were prepared exhibited higher $\mathrm{CV}$ areas than commercial carbon (Vulcan XC-72) due to the higher surface areas of the ACs. In particular, surface area influences AC capacitance greatly, because the activated sites for electron adsorption are also increased [22]. Therefore, most ACs subjected to chemical activation and acid washing processes had enhanced the surface areas, in addition to increased capacitance. Furthermore, the ACs treated with acids exhibited the visible redox peaks centred at approximately $0.42 \mathrm{~V}$ (oxidation) and $0.3 \mathrm{~V}$ (reduction).

Fig. 2d illustrates the capacitance calculated at different scan rates. However, in the present study, the $\mathrm{C}-\mathrm{SAC}-\mathrm{HCl}$ surface area was the highest, although its capacitance was not the highest. The capacitance of C-SAC- $\mathrm{H}_{3} \mathrm{PO}_{4}$ was higher than those of the other SACs. The relationship between the SSA and capacitance is presented in Fig. S3. The SAC and H-SAC exhibited the relatively low surface areas, which led a)

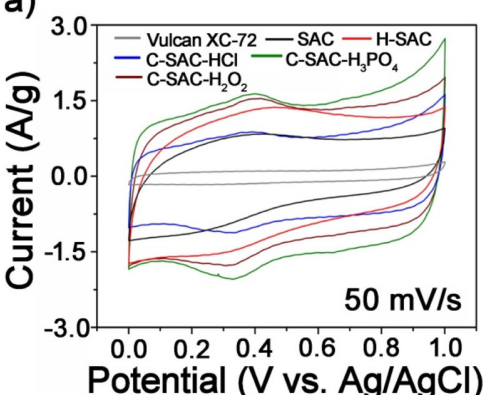

c)

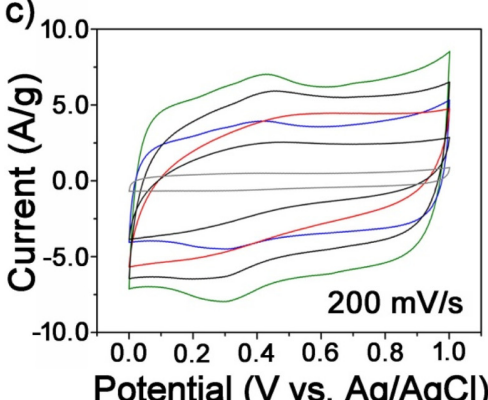

b)
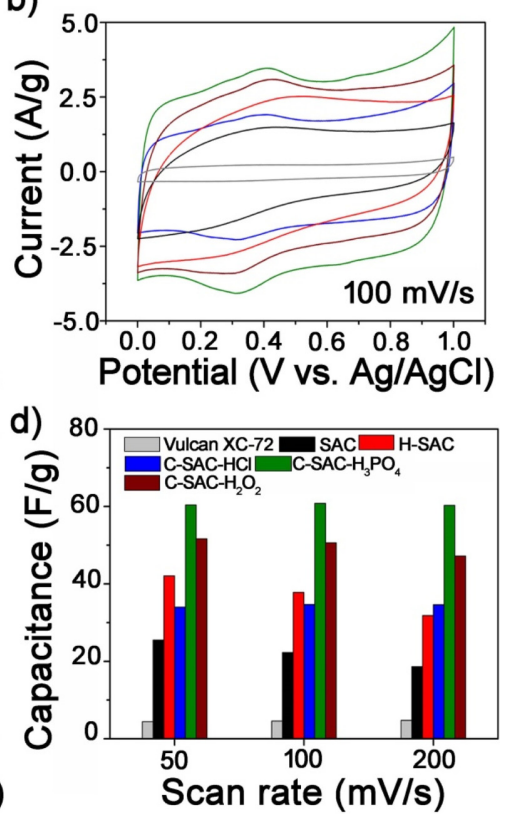

Fig. 3. Cyclic voltammetry of SAC, H-SAC, C-SAC-HCl, C-SAC- $\mathrm{H}_{3} \mathrm{PO}_{4}$, and C-SAC- $\mathrm{H}_{2} \mathrm{O}_{2}$ at 50 (a), 100 (b), and 200 (c) $\mathrm{mV} / \mathrm{s}$ scan rates, and their calculated capacitance comparison $(\mathrm{d})$. 
to lower capacitance. In addition, both C-SAC$\mathrm{H}_{3} \mathrm{PO}_{4}$ and $\mathrm{C}-\mathrm{SAC}-\mathrm{H}_{2} \mathrm{O}_{2}$ had higher surface areas, and exhibited higher capacitance. Conversely, the capacitance of C-SAC-HCl was lower than those of C-SAC- $\mathrm{H}_{3} \mathrm{PO}_{4}$ and C-SAC- $\mathrm{H}_{2} \mathrm{O}_{2}$, even though C$\mathrm{SAC}-\mathrm{HCl}$ had the highest surface area $\left(1637 \mathrm{~m}^{2} / \mathrm{g}\right)$. As aforementioned, treatment of C-SACs with various acids increased surface area; however, there was no relationship between surface area and capacitance. For example, although the surface area of C-SAC treated with the mild acid, $\mathrm{H}_{3} \mathrm{PO}_{4}$, was not the highest, it had the highest capacitance.

The mismatched and unexpected results could be due to surface functional groups. Functional groups react with electrons and influence capacitance, and are termed pseudo-capacitors. To validate the results, the measured $\mathrm{CV}$ areas were classified into two groups based on the storage types: electrical double layer capacitor (EDLC) and pseudo-capacitors, as shown in Fig. S4. As mentioned above, the EDLC and pseudo-capacitor were highly correlated with the surface areas and surface functional groups, respectively [23]. The red area inside CV area indicates EDLC, while the pink area represents pseudo-capacitor. In particular, redox peaks were detected between 0.2 and $0.6 \mathrm{~V}$ in the pseudo-capacitance areas. Redox peaks are caused by the oxidation of functional groups that are generated following acid treatments [24]. Conversely, sharp redox peaks were not observed in the H-SAC due to the elimination of surface functional groups following thermal treatment. The samples were analyzed by the X-ray photoelectron spectroscopy (XPS) to confirm the oxygen functional groups in Fig. S6 and Table S1. The number of oxygen functional groups was varied after thermal and chemical treatments of SACs. The Fig. S6a-e shows the XPS results of SAC, H-SAC, C-SAC-HCl, C-SAC- $\mathrm{H}_{3} \mathrm{PO}_{4}$, and C-SAC- $\mathrm{H}_{2} \mathrm{O}_{2}$. In addition, Fig. S6f shows the surface concentration of oxygen functional groups [3]. The SAC exhibited the higher oxygen concentrations due to the usage of water treatment process. In contrast, the concentration of thermal treatment of SAC (H-SAC) dramatically was dropped, causing by the removal of oxygen groups. The surface concentration increased again after the additional chemical treatment. Table $\mathrm{S} 1$ also shows the sum of oxygen functional groups. It indicated that the oxygen functional groups were increased after the chemical activation, and also affected the redox peaks as mentioned above (Fig. S4).

When compared with the other divided areas, the portion of ELDC out of the total capacitance was higher than that of the pseudo-capacitor, which indicated that surface area was the key factor influencing the total capacitance of samples. The amounts of functional groups could also be compared using elementary analysis (Fig. 4). Both oxygen and hydrogen concentrations decreased after thermal and chemical activation, as shown in Fig. 4b. In particular, the oxygen concentrations in C-SACs decreased lower than $10 \%$, which also indicated lower amounts of oxygen functional groups than in other SACs. The results indicate that EDLC area greatly influenced total capacitance.

Strong acids, in the present case, $\mathrm{HCl}$, efficiently removed ashes from the AC structures $[25,26]$. The carbon concentrations shown in Fig. 4 a were also consistent with the amount of ash removed. The concentrations increased considerably following treatment with $\mathrm{HCl}$, when compared with the other acids. Although the strong acid could remove the ashes efficiently, it could impair the AC structure [27]. Some
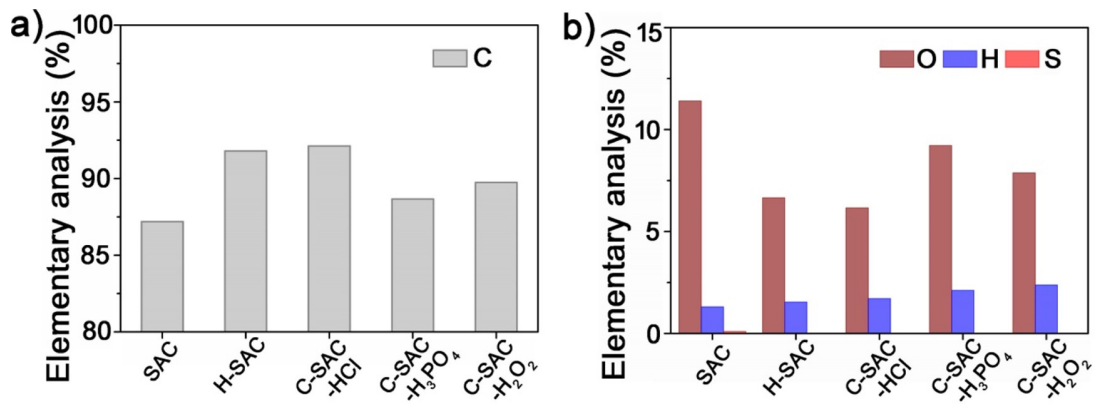

Fig. 4. Elementary analysis of $\mathrm{C}(\mathrm{a})$, and $\mathrm{O}, \mathrm{H}$, and $\mathrm{S}(\mathrm{b})$ in $\mathrm{SAC}, \mathrm{H}-\mathrm{SAC}, \mathrm{C}-\mathrm{SAC}-\mathrm{HCl}, \mathrm{C}-\mathrm{SAC}-\mathrm{H}_{3} \mathrm{PO}_{4}$, and C-SAC- $\mathrm{H}_{2} \mathrm{O}_{2}$. 


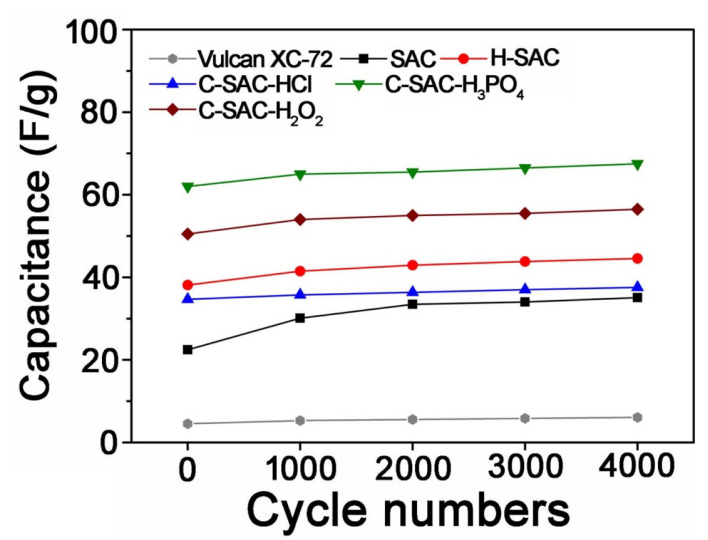

Fig. 5. Capacitance measurements for SAC, H-SAC, CSAC-HCl, C-SAC- $\mathrm{H}_{3} \mathrm{PO}_{4}$, and C-SAC- $\mathrm{H}_{2} \mathrm{O}_{2}$ at each $1000^{\text {th }}$ cycle at a scan rate of $100 \mathrm{mV} / \mathrm{s}$.

previous studies have already explored AC stability following acid treatment, and ACs treated with $\mathrm{HCl}$ exhibited poorer thermal stability than ACs treated with other acids such as $\mathrm{HNO}_{3}$ and $\mathrm{HF}$ [28].

Strong acids erode carbon skeletal structures, and, in turn, decrease their stability. Less stable C-SAC structures could negatively affect their capacitance. Furthermore, washing ACs with $\mathrm{H}_{3} \mathrm{PO}_{4}$ modifies their surfaces by increasing oxygen concentrations, which increases capacitance [29]. The presence of oxygenated groups could generate capacitance from quick Faradaic charge transfer reactions (pseudocapacitance) and electrostatic charging [30]. The contribution of pseudo-capacitance was the highest for $\mathrm{C}-\mathrm{SAC}-\mathrm{H}_{3} \mathrm{PO}_{4} \mathrm{~S}$ at any voltage window, which reflected the higher oxygen concentrations in $\mathrm{C}$ $\mathrm{SAC}-\mathrm{H}_{3} \mathrm{PO}_{4}$ when compared with the other samples treated with other acids, as shown in Fig. 4b. C-SAC$\mathrm{H}_{3} \mathrm{PO}_{4}$ has significantly higher $\mathrm{CO}$-evolving group contents, which are traditionally associated with quinone/hydroquinone-like redox activity $[31,32]$.

The electrochemical stability of samples was measured using continuous CV cycling tests as shown in Fig. S5. Fig. 5 shows the specific capacitance based on the CV curves at the $1 \mathrm{st}, 1000^{\text {th }}, 2000^{\text {th }}, 3000^{\text {th }}$, and $4000^{\text {th }}$ cycles. The capacitance at the $1000^{\text {th }}$ cycle was slightly higher when compared with the initial capacitance due to their activation, and then it remained constant. The capacitance did not change after the $4000^{\text {th }}$ cycle, and even the capacitance increased in C-SAC- $\mathrm{H}_{3} \mathrm{PO}_{4}$ and C-SAC- $\mathrm{H}_{2} \mathrm{O}_{2}$, which indicated that the ACs regenerated using the thermal and chemical activation methods and subsequently washed with mild acids, exhibited higher cyclic stability, and they could be used as capacitors. The results in Fig. S7 also indicate that the regenerated ACs could be used as capacitors. The capacitance observed in the study was approximately $60 \mathrm{~F} / \mathrm{g}$ at a scan rate of $100 \mathrm{mV} / \mathrm{s}$, which was similar or even higher than the capacitance reported in the previous studies [33-37].

\section{Conclusions}

SACs, which are often landfilled, were regenerated by thermal and chemical activation in sequence, and then applied in capacitors. In the present study, both activation and washing processes were performed. The regeneration processes, converted adsorbates into ashes, which remained in pores and blocked the pores. Pore blockage decreased specific surface area, and limited their applications. Consequently, the generated ashes were removed by washing with acids, namely $\mathrm{HCl}, \mathrm{H}_{3} \mathrm{PO}_{4}$, and $\mathrm{H}_{2} \mathrm{O}_{2}$. The strong acid $(\mathrm{HCl})$ removed the ashes efficiently, exhibited by highest specific surface area (C-SAC-HCl). However, C-SAC- $\mathrm{H}_{3} \mathrm{PO}_{4}$ and C-SAC- $\mathrm{H}_{2} \mathrm{O}_{2}$, which were treated with mild and weak acids, respectively, exhibited higher capacitance than C-SAC-HCl. Nevertheless, C-SAC-HCl, which exhibited the highest surface area, did not exhibit the highest capacitance, due to its less stable structure. The strong acid facilitated the removal of ashes from the pores, but it may have affected surface structures. In addition, $\mathrm{H}_{3} \mathrm{PO}_{4}$ oxidised AC surfaces and generated oxygen functional groups, leading to the higher pseudo-capacitance. Both ash removal and functional group generation in the regenerated ACs highly influenced their electrochemical performance. Regenerated AC stability was also tested using continuous $\mathrm{CV}$ cycling tests. The CV cycles were performed over 4000 cycles; however, C-SAC capacitance was not altered, leading to the high stability observed.

\section{Acknowledgment}

This work was supported by the Technology Innovation Program (20013038, Development of nonignition pitch based porous material technology for high adsorption of evaporative emissions) funded By 
the Ministry of Trade, Industry \& Energy (MI, Korea).

\section{Supporting Information}

Supporting Information is available at https:// doi.org/10.33961/jecst.2020.01683

\section{References}

[1] M.N. Nasruddin, M.R. Fahmi, C.Z.A. Abidin, T.S. Yen, J. Phys. Conf. Ser, 2018, 1116(3), 032022.

[2] P.-J. Lu, H.-C. Lin, W.-T. Yu, J.-M. Chern, J. Taiwan Inst. Chem. Eng., 2011, 42(2), 305-311.

[3] J.E. Park, G.B. Lee, B.U. Hong, S.Y. Hwang, Appl. Sci., 2019, 9(23), 5132.

[4] E. Sabio, E. González, J. González, C. González-García, A. Ramiro, J. Gañan, Carbon, 2004, 42(11), 2285-2293.

[5] D. Bhagawan, S. Poodari, G. Ravi kumar, S. Golla, C. Anand, K.S. Banda, V. Himabindu, S. Vidyavathi, $J$. Mater. Cycles Waste Manag., 2015, 17(1), 185-193.

[6] S.W. Nahm, W.G. Shim, Y.-K. Park, S.C. Kim, Chem Eng. J., 2012, 210, 500-509.

[7] A.L. Cazetta, O.P. Junior, A.M.M. Vargas, A.P. da Silva, X. Zou, T. Asefa, V.C. Almeida, J. Anal. Appl. Pyrolysis, 2013, 101, 53-60.

[8] Y. Guo, E. Du, Energy Procedia, 2012, 17, 444-449.

[9] E. Çalışkan, J.M. Bermúdez, J.B. Parra, J.A. Menéndez, M. Mahramanlığlu, C.O. Ania, J. Environ. Manage., 2012, 102, 134-140.

[10] R. Berenguer, J.P. Marco-Lozar, C. Quijada, D. CazorlaAmorós, E. Morallón, Energy \& Fuels, 2010, 24(6), 3366-3372.

[11] J.-Y. So, H.-R. Cho, Nucl. Eng. Technol., 2017, 49(4), 873-880.

[12] S.-G. Kim, H.-J. Son, J.-M. Jung, D.-C. Ryu, P.-J. Yoo, J. Environ. Sci. Int., 2015, 24(9), 1163-1170.

[13] X. Han, E. Wishart, Y. Zheng, Can. J. Chem. Eng., 2014, 92(5), 884-891.

[14] A. Bagreev, H. Rahman, T.J. Bandosz, Carbon, 2001, 39(9), 1319-1326.

[15] G.B. Lee, J.E. Park, S.Y. Hwang, J.H. Kim, S. Kim, H. Kim, B.U. Hong, Carbon Lett., 2019, 29(3), 263-272.

[16] C.-H. Lee, H.-N. Park, Y.-K. Lee, Y.S. Chung, S. Lee, H.-I. Joh, Electrochem. Commun., 2019, 106, 106516.

[17] K.P. Gable, Adv. Organomet. Chem, 1997, 41, 127-161.
[18] P.W. Atkins, Physical Chemistry, Oxford University Press, pp1024(data section), 1997.

[19] V.K. Chaudhri, B.E. John, S. Mishra, J. Pacheco, B. Porter, A. Spaulding, K-CAP'07: Proceedings of the Fourth International Conference on Knowledge Capture, 2007, 159-166.

[20] H.-I. Joh, H.K. Song, C.-H. Lee, J.-M. Yun, S.M. Jo, S. Lee, S.-I. Na, A.-T. Chien, S. Kumar, Carbon, 2014, 70, 308-312.

[21] L. Qie, W. Chen, H. Xu, X. Xiong, Y. Jiang, F. Zou, X. Hu, Y. Xin, Z. Zhang, Y. Huang, Energy Environ. Sci., 2013, 6(8), 2497-2504.

[22] T.E. Kibona, Appl. Sci., 2019, 1(9), 1-11.

[23] D. Kim, K. Lee, M. Kim, Y. Kim, H. Lee, Electrochim. Acta., 2019, 300, 461-469.

[24] J.-J. Yang, Y.-J. Choi, H.-J. Kim, Y.-J. Yuk, S.-G. Park, J. Korean Electrochem. Soc., 2013, 16(2), 65-69.

[25] J. Shah, M.R. Jan, F. Mabood, M. Shahid, J. Chinese Chem. Soc., 2006, 53(5), 1085-1089.

[26] S. Ouyang, D. Xiong, Y. Xie, L. Ma, Z. Hao, B. Sun, J. Yang, W. Chen, X. Li, Energy Sources, Part A Recover. Util. Environ. Eff., 2019, 1-17.

[27] S.-H. Pak, M.-J. Jeon, Y.-W. Jeon, Int. Biodeterior. Biodegradation, 2016, 113, 195-200.

[28] S. Wang, Z. Zhu, Dye. Pigment, 2007, 75(2), 306-314.

[29] V. Ruiz, C. Blanco, E. Raymundo-Piñero, V. Khomenko, F. Béguin, R. Santamaría, Electrochim. Acta., 2007, 52(4969), 90.

[30] B. E. Conway, Electrochemical Supercapacitors: Scientific Fundamentals and Technological Applications., 1999.

[31] M. Uchimiya, A.T. Stone, Geochim. Cosmochim. Acta., 2006, 70(6), 1388-1401.

[32] M. Quan, D. Sanchez, M.F. Wasylkiw, D.K. Smith, J. Am. Chem. Soc., 2007, 129(42), 12847-12856.

[33] K. Chaitra, R. T. Vinny, P. Sivaraman, N. Reddy, C. Hu, K. Venkatesh, C. S. Vivek, N. Nagaraju, N. Kathyayini, J. Energy Chem., 2017, 26(1), 56-62.

[34] V. Subramanian, C. Luo, A.M. Stephan, K.S. Nahm, S. Thomas, B. Wei, J. Phys. Chem. C, 2007, 111(20), 75277531.

[35] C. Kim, J.-W. Lee, J.-H. Kim, K.-S. Yang, Korean J. Chem. Eng., 2006, 23(4), 592-594.

[36] C.-S. Yang, Y.S. Jang, H.K. Jeong, Curr. Appl. Phys., 2014, 14(12), 1616-1620.

[37] S. Kumagai, M. Sato, D. Tashima, Electrochim. Acta., 2013, 114, 617-626. 